\title{
Propagation Mechanism Modeling in the Near-Region of Arbitrary Cross-Sectional Tunnels
}

\author{
Ke Guan, ${ }^{1}$ Zhangdui Zhong, ${ }^{1}$ Bo Ai, ${ }^{1}$ Ruisi He, ${ }^{1}$ Yuanxuan Li, ${ }^{1}$ and César Briso Rodríguez ${ }^{2}$ \\ ${ }^{1}$ State Key Laboratory of Rail Traffic Control and Safety, Beijing Jiaotong University, Beijing 100044, China \\ ${ }^{2}$ Escuela Universitaria de Ingeniería Técnica de Telecomunicación, Universidad Politècnica de Madrid, 28031 Madrid, Spain
}

Correspondence should be addressed to Ke Guan, myecone@hotmail.com

Received 24 April 2012; Accepted 24 September 2012

Academic Editor: Thomas Kürner

Copyright $(2012 \mathrm{Ke}$ Guan et al. This is an open access article distributed under the Creative Commons Attribution License, which permits unrestricted use, distribution, and reproduction in any medium, provided the original work is properly cited.

\begin{abstract}
Along with the increase of the use of working frequencies in advanced radio communication systems, the near-region inside tunnels lengthens considerably and even occupies the whole propagation cell or the entire length of some short tunnels. This paper analytically models the propagation mechanisms and their dividing point in the near-region of arbitrary cross-sectional tunnels for the first time. To begin with, the propagation losses owing to the free space mechanism and the multimode waveguide mechanism are modeled, respectively. Then, by conjunctively employing the propagation theory and the three-dimensional solid geometry, the paper presents a general model for the dividing point between two propagation mechanisms. It is worthy to mention that this model can be applied in arbitrary cross-sectional tunnels. Furthermore, the general dividing point model is specified in rectangular, circular, and arched tunnels, respectively. Five groups of measurements are used to justify the model in different tunnels at different frequencies. Finally, in order to facilitate the use of the model, simplified analytical solutions for the dividing point in five specific application situations are derived. The results in this paper could help deepen the insight into the propagation mechanisms in tunnels.
\end{abstract}

\section{Introduction}

Effective prediction models of propagation properties in tunnels are highly requested in the design and planning phases of advanced radio systems. In order to describe the propagation characteristics inside tunnels, most models presented in the last four decades indicate that there is a "critical distance" $[1,2]$, normally called the break point [13]. Before the break point is the near-region, where the highorder modes are significant; guided propagation has not been well established, and, therefore, the signal suffers larger loss. After the break point is the far-region, where the high order modes have been greatly attenuated; guided propagation has been stabilized and undergoes a smaller loss $[2,4-6]$.

The distance from the transmitter to the break point is expressed by [1]

$$
Z_{N R}=\operatorname{Max}\left(\frac{W^{2}}{\lambda}, \frac{H^{2}}{\lambda}\right)
$$

where $Z_{N R}, W, H$, and $\lambda$ denote the length of near-region, the width and the height of rectangular tunnel, as well as the signal wavelength in metres. This formula can be applied in arched and circular tunnel cases as the EM field distribution and attenuation of the modes in arched and circular tunnels are almost the same as the rectangular tunnel [7]. Please note that $Z_{N R}$ is inversely proportional to the wavelength.

By making a review on the development of wireless communication systems, we can find that the near-region inside tunnels lengthens greatly resulting from the increase of working frequencies. In the public communication field, the representative systems can be listed as GSM (Global System for Mobile Communications), 3G (3rd Generation), WiFi (Wireless Fidelity), and WiMAX (World Interoperability for Microwave Access). Their frequencies are $900 / 1800 \mathrm{MHz}$, $2 \mathrm{GHz}, 2.4 / 5 \mathrm{GHz}, 3.8 \mathrm{GHz}, 5.7 \mathrm{GHz}$, and $5.8 \mathrm{GHz}$, respectively. By assuming the maximum of the width and the height of the equivalent rectangular tunnel to be $15 \mathrm{~m}$, which is very common for the double-track subway tunnel 
or railway tunnel, (1) calculates the length of the nearregion in each system as $675 / 1350 \mathrm{~m}, 1500 \mathrm{~m}, 1800 / 3750 \mathrm{~m}$, $2850 \mathrm{~m}, 4275 \mathrm{~m}$, and $4350 \mathrm{~m}$, respectively. In the dedicated communication area, the list could be TETRA (Terrestrial Trunked Radio) [8], GSM-R (Global System for Mobile Communications for Railway) [9], CBTC (CommunicationBased Train Control System) [10], and DSRC (Dedicated Short-range Communications) [11]. Their frequencies are $400 \mathrm{MHz}, 900 \mathrm{MHz}, 2.4 \mathrm{GHz}$, and $5.8 / 5.9 \mathrm{GHz}$, respectively. The corresponding near-regions can be $300 \mathrm{~m}, 675 \mathrm{~m}$, $1800 \mathrm{~m}$, and $4350 / 4425 \mathrm{~m}$, respectively. This great change reveals the fact that the near-region may occupy most of the propagation cell at high frequencies. Especially in some short tunnels or high reliable systems that require a moderate overlapping of the transmitters, the whole propagation cell could even only be in the near-region. However, there is no unanimous consensus, yet there is on the propagation in the near-region. Some researchers are inclined to interpret the propagation before the break point with the single ray (free space) theory $[6,12,13]$, whereas others contend that it should be described by the multimode waveguide model $[1,2,14]$. In fact, a big deal of evidence proves that the free space mechanism should be established firstly and the multimode propagation mechanism comes later. Thus, in order to clearly reveal the propagation mechanism situation in the near-region, it is essential to model the accurate location of the dividing point between the two mechanisms. This paper presents a novel general analytical model that can be employed in arbitrary cross-sectional tunnels.

\section{Modeling for the Propagation Mechanisms and Their Dividing Point}

2.1. Geometrical Modeling for Rectangular, Circular, and Arched Tunnels. Generally speaking, rectangular, circular, and arched tunnels cover almost all the realistic application situations. An extensive comparison of theoretical and experimental results allowed us to show that the copolar field variation in an arched tunnel can be predicted with sufficient accuracy by using modal theory and assuming a rectangular tunnel [15]. Meanwhile, the EM field distribution and attenuation of the modes in circular waveguide are almost the same as the rectangle waveguide [16]. Hence, in the analysis of the propagation loss in the multi-mode waveguide segment, the tunnel's cross section is treated as an equivalent rectangle with a width of $w$ and a height of $h$. A Cartesian coordinate system is set with its origin located at the center of the rectangle tunnel.

In the case of an arched tunnel, the size of the equivalent rectangular waveguide that is used in the model can be computed by taking the main horizontal dimension $h$ close to the tunnel's floor size and computing the vertical dimension $w$ using the "rule of thumb", that is,

$$
h=\sqrt{4 R^{2}-w^{2}},
$$

where $R$ is the radius of the arched-ceiling/wall's circle. This idealized geometry is common in modern road and railway tunnels [17]. In the case of a circular tunnel, $w=h=2 R$, where $R$ is the radius of the radius of the cross-sectional circle.

\subsection{Propagation Loss in Different Propagation Mechanisms}

2.2.1. Propagation Loss in the Free Space Propagation Segment. In the adjacent region of the transmitter antenna, the angles of incidence from the ray to the wall (vertical, horizontal, and circular) are high resulting in high attenuation of reflected rays, whereas the path difference between direct and reflected rays may also cause additional attenuation; thus, only the direct ray significantly contributes to the strength of the received signal. The channel loss in this segment follows the free space loss attenuation [18]

$$
P L(d B)=-10 \log _{10}\left[\frac{\lambda^{2}}{(4 \pi)^{2}\left|z_{r}-z_{t}\right|^{2}}\right],
$$

where $\left|z_{r}-z_{t}\right|$ is the distance between the transmitter and receiver in meters and $\lambda$ is the signal wavelength.

\subsubsection{Propagation Loss in the Multimode Waveguide Segment.} According to the modal theory, an equivalent rectangular tunnel can be regarded as an oversized imperfect hollow rectangular waveguide. Since the UHF is much higher than the cutoff frequency of the fundamental modes which is very low, a wide range of $E_{m n}$ multiple modes propagate when the free space segment ends [12].

By employing the modal theory, the general expression of the attenuation constant with horizontally and vertically polarized $E_{m n}$ modes inside various tunnels [19], such as circular tunnel, rectangular tunnel, arched tunnel, oval tunnel, and so forth can be given by

$$
\begin{aligned}
& \alpha(m, n)^{h}=\varphi \lambda^{2}\left(\frac{m^{2} \varepsilon_{r}}{w^{3} \sqrt{\varepsilon_{r}-1}}+\frac{n^{2}}{h^{3} \sqrt{\varepsilon_{r}-1}}\right) \frac{d B}{m}, \\
& \alpha(m, n)^{v}=\varphi \lambda^{2}\left(\frac{m^{2}}{w^{3} \sqrt{\varepsilon_{r}-1}}+\frac{n^{2} \varepsilon_{r}}{h^{3} \sqrt{\varepsilon_{r}-1}}\right) \frac{d B}{m},
\end{aligned}
$$

where $w$ and $h$ denote the maximum of the width and the height, respectively; $\varphi$ is a coefficient, its value varies by the different shape of the tunnel [20]: rectangular tunnel, $\varphi=$ 4.343; circular tunnel, $\varphi=5.09$; arched tunnel, $\varphi=5.13$. $\varepsilon_{v}$ and $\varepsilon_{h}$ are relative permittivity for vertical and horizontal walls, with the typical values for concrete: $\varepsilon_{v}=\varepsilon_{h}=5$ [1].

Besides the geometry of the tunnel, the roughness of walls of the tunnel influences the propagation loss as well. Hence, the attenuation owing to the roughness introduced by [2] is involved in the model. Finally, the propagation loss in the multi-mode waveguide segment can be obtained by considering both the polarizations and the roughness of walls;

$$
\begin{gathered}
L_{m n}^{v / h}(d B)=10 \log _{10}\left[\sum_{i=1}^{m} \sum_{j=1}^{n} \times \sqrt{10^{2 \alpha(i, j)^{h}}\left|z_{r}-z_{t}\right|}+10^{2 \alpha(i, j)^{v}\left|z_{r}-z_{t}\right|}\right] \\
+8.686 \pi^{2} \gamma^{2} \lambda\left(\frac{1}{w^{4}}+\frac{1}{h^{4}}\right)\left|z_{r}-z_{t}\right|
\end{gathered}
$$

where $\gamma$ is the root-mean-square roughness. 


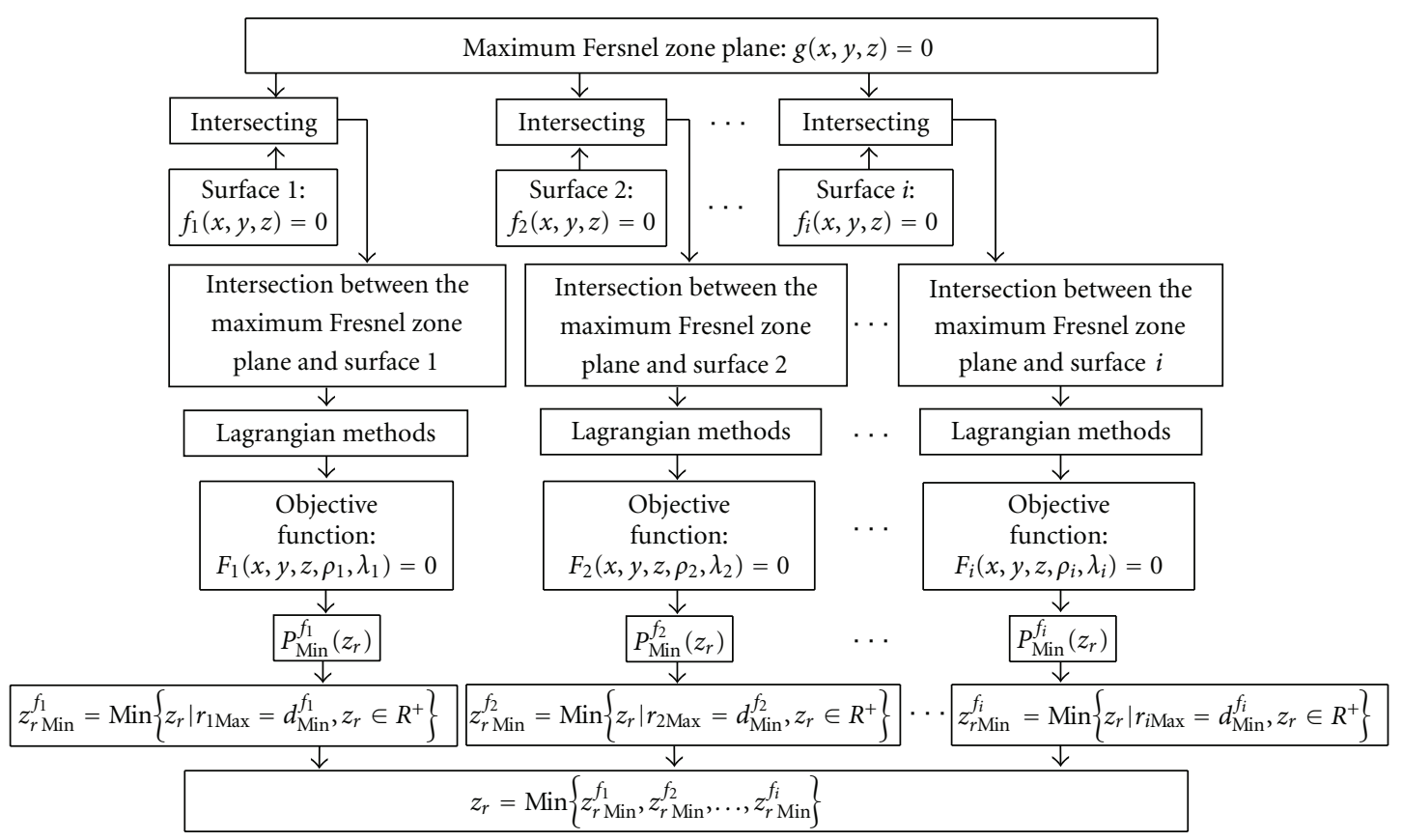

FIGURE 1: Flow chart of modeling for the diving point in arbitrary cross-sectional tunnels.

2.3. Modeling for Dividing Point between Different Propagation Mechanisms in Arbitrary Cross-Sectional Tunnels. In order to clarify the propagation mechanism situation, it is necessary to model accurately the location of the dividing point between the free space propagation segment and the multi-mode waveguide segment.

On the basis of the analysis of the propagation procedure inside tunnels, it can be known that the point where the first Fresnel zone is tangent to the walls of the tunnel is the dividing point between the two mechanisms. However, the localization of it is not an easy work. Since the interaction between the first Fresnel zone and the walls depends on a large number of factors, such as the locations of the transmitter and receiver, the dimensions of the tunnel, the working frequency, the computational time would be intolerable if all the elements were considered when we track the interaction and its change law. Thus, it is desirable to find a simple parameter representing the interaction.

According to the geometry, it is easy to determine the distance between the tangent line/curve (of the maximum Fresnel zone plate and the walls) and the middle point (of the line of sight between transmitter and receiver). If this distance is larger than the radius of the maximum first Fresnel zone plate, the first Fresnel zone can be treated as almost clear. We have to admit that in this case some parts inside the first Fresnel zone could still be blocked. But since the first Fresnel zone is a flat ellipsoid, such kind of slight obstruction does not result in many effective reflected rays or obvious diffractive loss. Hence, the free space propagation model can still work. When this distance is smaller than the radius, which means even the widest part of the first Fresnel zone is blocked, more severe obstruction occurs in the other parts. Thus, the relative relation between this distance and the radius can be employed to reflect the interaction between the first Fresnel zone and the walls to some extent. Furthermore, the location of the dividing point can be deduced when the distance and the radius are equal.

Figure 1 illustrates the flow chart of the concrete modeling process. The first step is to geometrically model the arbitrary cross-sectional tunnel and all the relative components. Figure 2 depicts the three-dimensional geometry schematic diagram of an arbitrary cross-sectional tunnel, transmitter, receiver, line of sight, and the maximum first Fresnel zone.

According to the three-dimensional solid geometry, the arbitrary cross-sectional tunnel consists of a set of plane surfaces and curved surfaces whose coordinates $x, y$, and $z$ satisfy the following equation

$$
f_{i}(x, y, z)=0, \quad i=1,2, \ldots, n .
$$

The coordinates of transmitter, receiver, and the middle point on the line of sight between transmitter and receiver are $P_{t}\left(x_{t}, y_{t}, z_{t}\right), P_{r}\left(x_{r}, y_{r}, z_{r}\right)$, and $P_{0}\left(x_{0}, y_{0}, z_{0}\right)$; their relationships are expressed by

$$
x_{0}=\frac{x_{r}+x_{t}}{2}, \quad y_{0}=\frac{y_{r}+y_{t}}{2}, \quad z_{0}=\frac{z_{r}+z_{t}}{2} .
$$

Then, the maximum Fresnel zone plane can be expressed by a plane in general type

$$
\begin{gathered}
\left(x_{r}-x_{t}\right)\left(x-\frac{x_{r}+x_{t}}{2}\right)+\left(y_{r}-y_{t}\right)\left(y-\frac{y_{r}+y_{t}}{2}\right) \\
+\left(z_{r}-z_{t}\right)\left(z-\frac{z_{r}+z_{t}}{2}\right)=0 .
\end{gathered}
$$




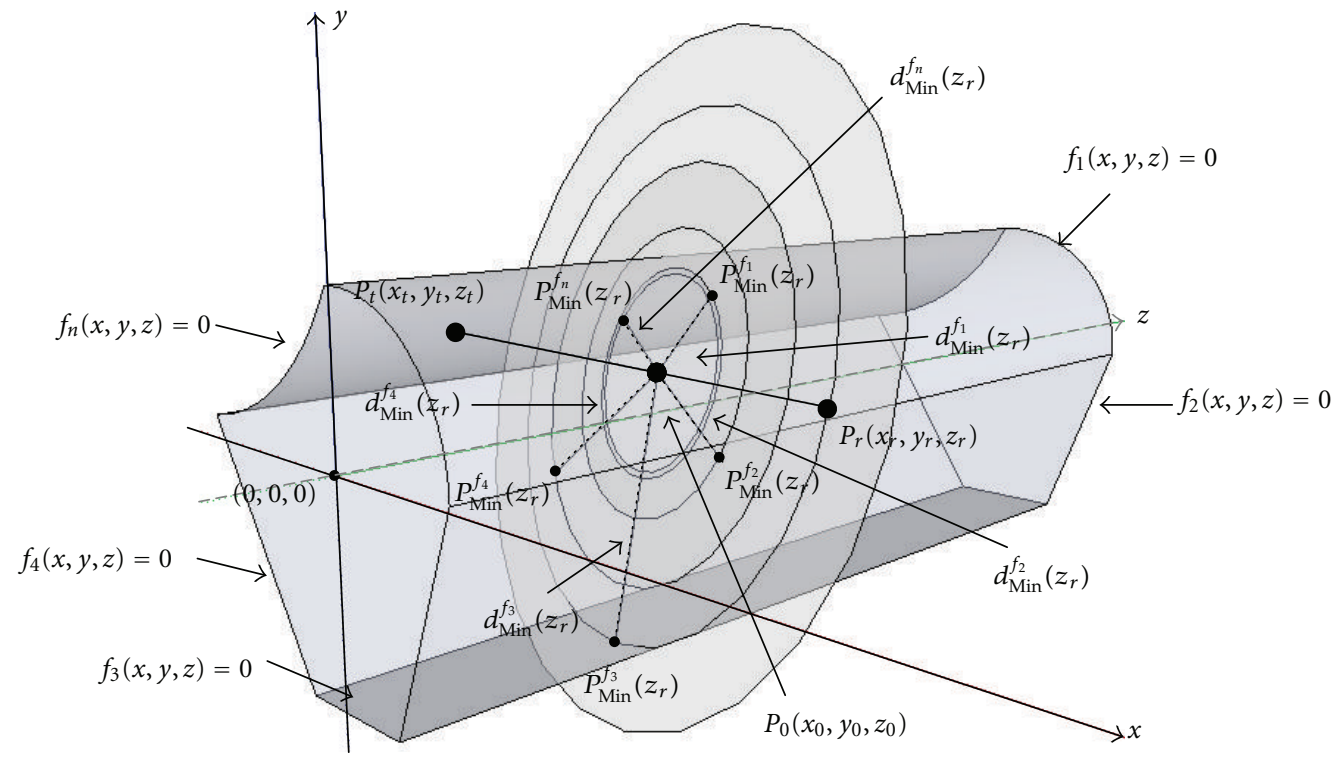

FIgURE 2: Detailed schematic diagram of the propagation inside arbitrary cross-sectional tunnels with the first Fresnel zone clearance.

Thus, the intersection between the maximum Fresnel zone plane and the surface $f_{i}$ of the tunnel is a curve or a line which can be written by

$$
\begin{gathered}
\left(x_{r}-x_{t}\right)\left(x-\frac{x_{r}+x_{t}}{2}\right)+\left(y_{r}-y_{t}\right)\left(y-\frac{y_{r}+y_{t}}{2}\right) \\
+\left(z_{r}-z_{t}\right)\left(z-\frac{z_{r}+z_{t}}{2}\right)=0, \\
f_{i}(x, y, z)=0, \quad i=1,2, \ldots, n .
\end{gathered}
$$

Define the first equation as a function $g(x, y, z)$ by

$$
\begin{aligned}
g(x, y, z)= & \left(x_{r}-x_{t}\right)\left(x-\frac{x_{r}+x_{t}}{2}\right) \\
& +\left(y_{r}-y_{t}\right)\left(y-\frac{y_{r}+y_{t}}{2}\right) \\
& +\left(z_{r}-z_{t}\right)\left(z-\frac{z_{r}+z_{t}}{2}\right) .
\end{aligned}
$$

Define the second equation as a function $f_{i}(x, y, z)$.

In order to find the minimal distance between the intersection (line/curve) and the middle point $P_{0}\left(x_{0}, y_{0}, z_{0}\right)$ on the line of sight, the Lagrange multiplier method seeking extremum is employed. Construct a function as follows:

$$
\begin{aligned}
F_{i}\left(x, y, z, \rho_{i}, \lambda_{i}\right)= & \left(x-\frac{x_{r}+x_{t}}{2}\right)^{2}+\left(y-\frac{y_{r}+y_{t}}{2}\right)^{2} \\
& +\left(z-\frac{z_{r}+z_{t}}{2}\right)^{2}+\rho_{i} \cdot g(x, y, z) \\
& +\lambda_{i} \cdot f_{i}(x, y, z),
\end{aligned}
$$

where $\xi$, and $\mu$ are the Lagrange multipliers. By seeking partial derivative of $x, y$, and $z$, respectively, (11) can be transformed to

$$
\begin{gathered}
\frac{\partial F_{i}}{\partial x}=2\left(x-\frac{x_{r}+x_{t}}{2}\right)+\rho_{i} \cdot\left(x_{r}-x_{t}\right)+\lambda_{i} \cdot \frac{\partial f_{i}}{\partial x}=0, \\
\frac{\partial F_{i}}{\partial y}=2\left(y-\frac{y_{r}+y_{t}}{2}\right)+\rho_{i} \cdot\left(y_{r}-y_{t}\right)+\lambda_{i} \cdot \frac{\partial f_{i}}{\partial y}=0, \\
\frac{\partial F_{i}}{\partial z}=2\left(z-\frac{z_{r}+z_{t}}{2}\right)+\rho_{i} \cdot\left(z_{r}-z_{t}\right)+\lambda_{i} \cdot \frac{\partial f_{i}}{\partial z}=0, \\
\frac{\partial F_{i}}{\partial \rho_{i}}=\left(x_{r}-x_{t}\right)\left(x-\frac{x_{r}+x_{t}}{2}\right)+\left(y_{r}-y_{t}\right)\left(y-\frac{y_{r}+y_{t}}{2}\right) \\
+\left(z_{r}-z_{t}\right)\left(z-\frac{z_{r}+z_{t}}{2}\right)=0, \\
\frac{\partial F_{i}}{\partial \lambda_{i}}=f_{i}=0 .
\end{gathered}
$$

By seeking the simultaneous solution of (12), the coordinate of intersection point with the minimal distance to $P_{0}\left(x_{0}, y_{0}, z_{0}\right)$ can be obtained: $p_{\text {Min }}^{f_{i}}\left(x^{f_{i}}\left(z_{r}\right), y^{f_{i}}\left(z_{r}\right), z^{f_{i}}\left(z_{r}\right)\right)$. Therefore, the minimal distance between $P_{0}$ and the intersection (line/curve) between the maximum Fresnel zone plane and the surface $f_{i}$ of the tunnel can be expressed as

$$
\begin{aligned}
d_{\text {Min }}^{f_{i}}\left(z_{r}\right)= & {\left[\left(x^{f_{i}}\left(z_{r}\right)-\frac{x_{r}+x_{t}}{2}\right)^{2}\right.} \\
& +\left(y^{f_{i}}\left(z_{r}\right)-\frac{y_{r}+y_{t}}{2}\right)^{2} \\
& \left.+\left(z^{f_{i}}\left(z_{r}\right)-\frac{z_{r}+z_{t}}{2}\right)^{2}\right]^{1 / 2} .
\end{aligned}
$$


On the basis of the propagation theory, the radius of the first Fresnel zone is determined by

$$
r_{1}=\sqrt{\frac{\lambda d_{1} d_{2}}{d_{1}+d_{2}}},
$$

where $d_{1}$ denotes the distance between the transmitter and the interaction between the line of sight and the first Fresnel zone, and $d_{2}$ denotes the distance between the receiver and the interaction. When the interaction is the middle point $P_{0}$, $d_{1}=d_{P_{t} P_{0}}=d_{2}=d_{P_{0} P_{r}}=(1 / 2) d_{P_{t} P_{r}}$. At this point, the radius gets the maximum value of the first Fresnel zone

$$
r_{1 \mathrm{Max}}\left(z_{r}\right)=\frac{1}{2} \sqrt{\lambda d_{P_{t} P_{r}}}
$$

The propagation theory indicates that the free space loss channel model can be applied if the first Fresnel zone is free of any obstacles. Therefore, if only the wall $f_{i}(x, y, z)$ of the tunnel could be touched by the maximum first Fresnel zone, the dividing point between two propagation mechanisms locates at $z_{r \text { Min }}^{f_{i}}$ which is the minimal positive real root of the

$$
r_{1 \mathrm{Max}}\left(z_{r}\right)=d_{\mathrm{Min}}^{f_{i}}\left(z_{r}\right)
$$

Hence, $z_{r \text { Min }}^{f_{i}}$ can be expressed by

$$
z_{r \operatorname{Min}}^{f_{i}}=\operatorname{Min}\left\{z_{r} \mid r_{i \operatorname{Max}}=d_{\mathrm{Min}}^{f_{i}}, z_{r} \in R^{+}\right\}
$$

which means the maximum first Fresnel zone first touches the surface $f_{i}(x, y, z)$ of tunnels.

However, in fact, there are totally $n$ walls of the arbitrary cross-sectional tunnels that could be tangent to the maximum first Fresnel zone. Therefore, the dividing point locates at $z_{r}$ when the maximum first Fresnel zone first touches any one of the walls. Thus, the dividing point locates at

$$
z_{r}=\operatorname{Min}\left\{z_{r \operatorname{Min}}^{f_{i}}, i=1,2, \ldots, n\right\},
$$

which means the maximum first Fresnel zone first touches any one of the surfaces of tunnels.

\section{Dividing Point Model Validation in Rectangular, Circular, and Arched Tunnels}

Theoretically, the general model can be employed in arbitrary cross-sectional tunnels by substituting various parameters. Here, we give the specific model and corresponding validation in the main types of tunnels in reality (rectangular, circular, and arched tunnels), respectively.

3.1. Dividing Point Model in Rectangular Tunnel. Figure 3 demonstrates the propagation inside a rectangular tunnel with the first Fresnel zone clearance. In a rectangular tunnel, two vertical walls and two horizontal planes can possibly obstruct the first Fresnel zone; thus, by substituting following functions:

(i) Left vertical wall: $f_{\text {Pla }-L}: x=-b$;

(ii) Right vertical wall: $f_{\text {Pla }-R}: x=b$;
TABLE 1: Comparisons of the dividing point between the model, and the measurements inside rectangular circular, and arched tunnels.

\begin{tabular}{lccc}
\hline Tunnel & $\begin{array}{c}\text { Frequency } \\
(\mathrm{GHz})\end{array}$ & $\begin{array}{c}\text { Measured } \\
\text { result }\end{array}$ & $\begin{array}{c}\text { Theoretical } \\
\text { prediction }\end{array}$ \\
\hline $\begin{array}{l}\text { Railway tunnel in } \\
\text { Spain [1] }\end{array}$ & 0.9 & $30-35 \mathrm{~m}$ & $30.86 \mathrm{~m}$ \\
$\begin{array}{l}\text { Vehicle tunnel in } \\
\text { France [21] }\end{array}$ & 0.45 & $35-40 \mathrm{~m}$ & $37.88 \mathrm{~m}$ \\
$\begin{array}{l}\text { Vehicle tunnel in } \\
\text { France [21] }\end{array}$ & 0.9 & $70-75 \mathrm{~m}$ & $75.76 \mathrm{~m}$ \\
$\begin{array}{l}\text { Pedestrian tunnel in } \\
\text { Europe [12] } \\
\text { Road tunnel } \\
\begin{array}{l}\text { Austria-Slovenia } \\
\text { [12] }\end{array}\end{array}$ & 0.4 & $15 \mathrm{~m}$ & $13.65 \mathrm{~m}$ \\
\hline
\end{tabular}

(iii) ceiling: $f_{\text {Pla-C }}: y=a$;

(iv) Floor: $f_{\mathrm{Pla}-F}: y=-c$;

to $(6), d_{\mathrm{Min}}^{\mathrm{Pla}-L}\left(z_{r}\right), d_{\mathrm{Min}}^{\mathrm{Pla}-R}\left(z_{r}\right), d_{\mathrm{Min}}^{\mathrm{Pla}-C}\left(z_{r}\right)$, and $d_{\mathrm{Min}}^{\mathrm{Pla}-F}\left(z_{r}\right)$ corresponding to the minimal distance from $P_{0}$ to the intersection line on the left vertical wall, right vertical wall, ceiling, and floor can be obtained. By using (16), the dividing point location of $z_{r \text { Min }}^{\text {Pla } L}, z_{r \text { Min }}^{\text {Pla- } R}, z_{r \text { Min }}^{\text {Pla } C}$, and $z_{r \text { Min }}^{\text {Pla- } F}$, corresponding to the touching of the maximum first Fresnel zone and the left wall, right wall, ceiling, and floor of rectangular tunnels, respectively, can be derived as

$$
\begin{aligned}
& z_{r \text { Min }}^{\mathrm{Pla}-L}=\operatorname{Min}\left\{z_{r} \mid r_{1 \mathrm{Max}}\left(z_{r}\right)=d_{\mathrm{Min}}^{\mathrm{Pla}-L}, z_{r} \in R^{+}\right\}, \\
& z_{r \text { Min }}^{\mathrm{Pla}-R}=\operatorname{Min}\left\{z_{r} \mid r_{1 \mathrm{Max}}\left(z_{r}\right)=d_{\mathrm{Min}}^{\mathrm{Pla}-R}, z_{r} \in R^{+}\right\}, \\
& z_{r \text { Min }}^{\mathrm{Pla}-C}=\operatorname{Min}\left\{z_{r} \mid r_{1 \mathrm{Max}}\left(z_{r}\right)=d_{\mathrm{Min}}^{\mathrm{Pla}-C}, z_{r} \in R^{+}\right\}, \\
& z_{r \mathrm{Min}}^{\mathrm{Pla}-F}=\operatorname{Min}\left\{z_{r} \mid r_{1 \mathrm{Max}}\left(z_{r}\right)=d_{\mathrm{Min}}^{\mathrm{Pla}-F}, z_{r} \in R^{+}\right\} .
\end{aligned}
$$

Then, the dividing point between two propagation mechanisms inside a rectangular tunnel locates at $z_{r}$ :

$$
z_{r}=\operatorname{Min}\left(z_{r \text { Min }}^{\text {Pla }-R}, z_{r \text { Min }}^{\text {Pla- } R}, z_{r \text { Min }}^{\text {Pla- }}, z_{r \text { Min }}^{\text {Pla- } F}\right)
$$

In order to validate the performance of the model in rectangular tunnels, a set of measurements is taken in one of the longest tunnels of the new $450 \mathrm{~km}$ high-speed train line from Madrid to Lleida in Spain [1]: $\lambda=0.33, a=3.15$, $b=5.35, c=3.15, x_{t}=-5.15, y_{t}=0.85, z_{t}=0$, $x_{r}=-2.35$, and $y_{r}=-0.15$. By seeking the minimal positive real root of the simultaneous solution of (12), the results are the coordinates of the intersection points in each surface with the minimal distance to $P_{0}\left(x_{0}, y_{0}, z_{0}\right)$ in the rectangular tunnel: $p_{\text {Min }}^{\text {Pla- }}(-5.35,0.35,15.58), p_{\text {Min }}^{\text {Pla- } R}(5.35,0.35,497.33)$, $p_{\text {Min }}^{\text {Pla } C}(-3.75,3.15,47.1)$, and $p_{\text {Min }}^{\text {Pla- } F}(-3.75,-3.15,73.54)$. By solving (16) and (17), $z_{r \text { Min }}^{\text {Pla- } L}=30.86, z_{r \text { Min }}^{\text {Pla } R}=994.72$, $z_{r \text { Min }}^{\text {Pla- }}=94.14$, and $z_{r \text { Min }}^{\text {Pla- }}=147.12$. Hence, according to (18), the dividing point locates at $z_{r}=30.86$ in this case. Comparison results are shown in Table 1. 


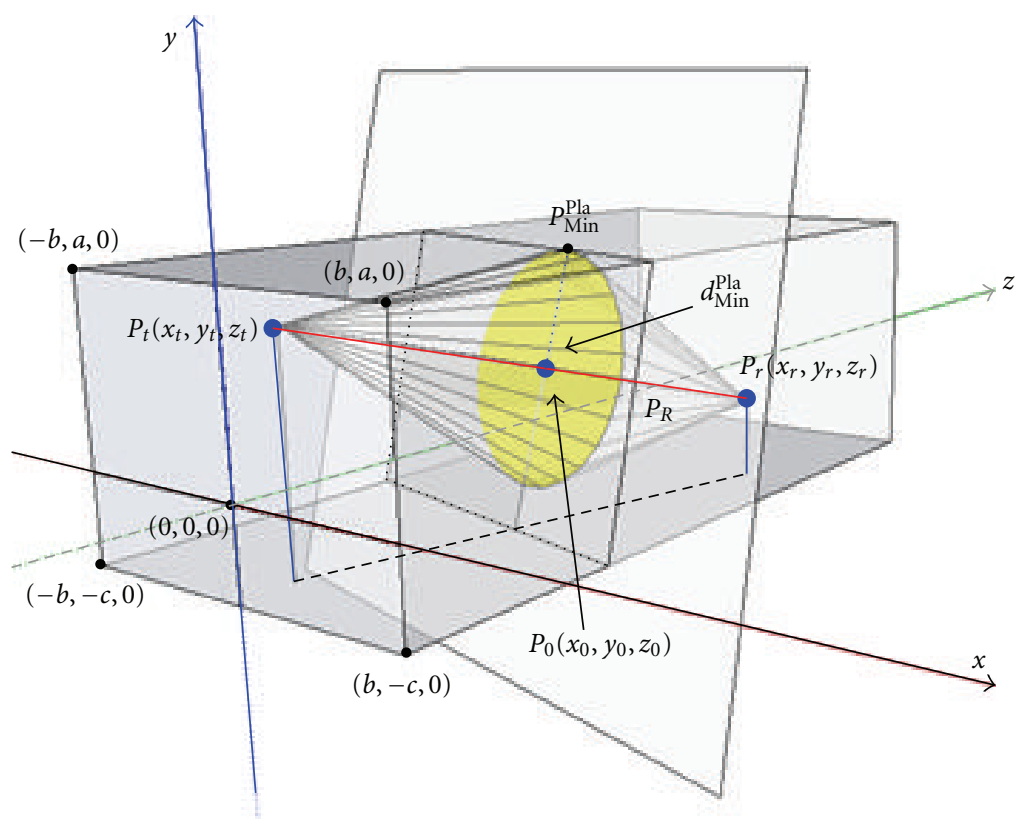

FIGURE 3: Detailed schematic diagram of the propagation inside rectangular tunnels with the first Fresnel zone clearance.

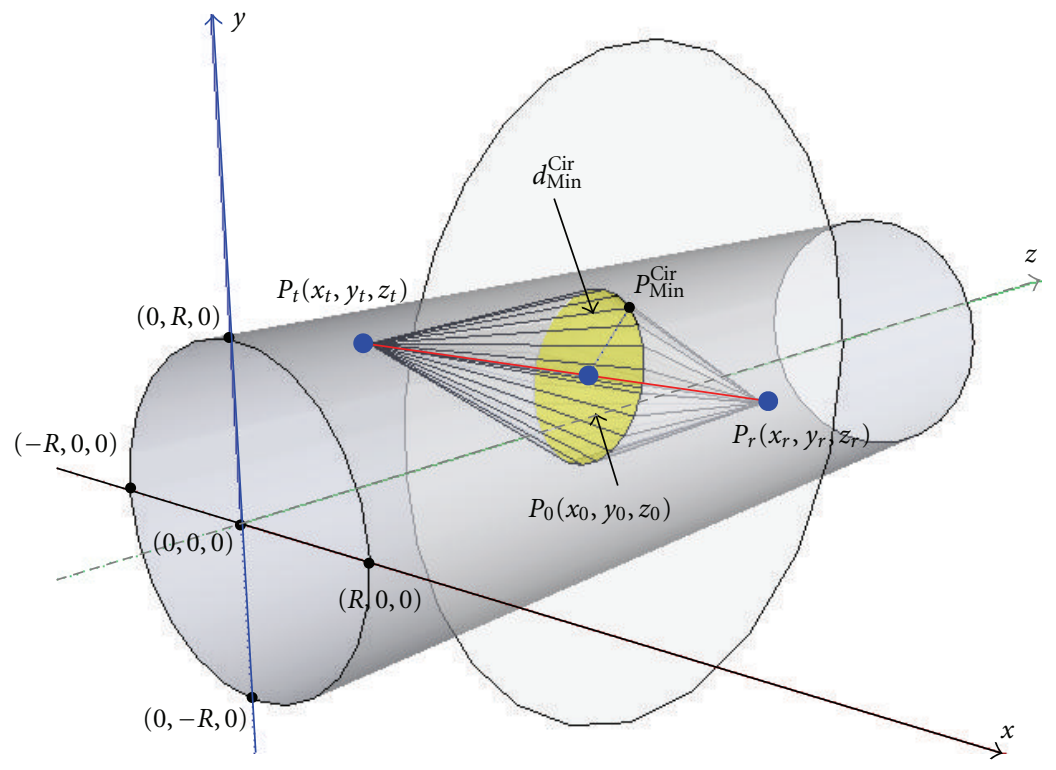

FIGURE 4: Detailed schematic diagram of the propagation inside circular tunnels with the first Fresnel zone clearance.

3.2. Dividing Point Model in Circular Tunnel. Figure 4 illustrates the propagation inside circular tunnel with the first Fresnel zone clearance. In circular tunnel, the circular walls can possibly obstruct the first Fresnel zone; thus, by substituting the following function:

(i) Circular wall: $f_{\text {Cir }}: x^{2}+y^{2}=R^{2}$ to $(6), d_{\text {Min }}^{\mathrm{Cir}}\left(z_{r}\right)$ corresponding to the minimal distance from $P_{0}$ to the intersection curve on the circular wall can be obtained. By using (16), the dividing point location of $z_{r \text { Min }}^{\mathrm{Cir}}$ corresponding to the touching of the maximum first Fresnel zone and the circular wall of tunnels can be obtained as

$$
z_{r \text { Min }}^{\mathrm{Cir}}=\operatorname{Min}\left\{z_{r} \mid r_{1 \mathrm{Max}}\left(z_{r}\right)=d_{\mathrm{Min}}^{\mathrm{Pla}-L}, z_{r} \in R^{+}\right\} .
$$

Then, the dividing point between mechanisms inside a circular tunnel locates at $z_{r}$ :

$$
z_{r}=\operatorname{Min}\left(z_{r \text { Min }}^{\mathrm{Cir}}\right)=z_{r \text { Min }}^{\mathrm{Cir}} .
$$

In order to validate the performance of the model in circular tunnels, two groups of experiments in a $3.5 \mathrm{~km}$-long straight tunnel in the Massif Central of south-central France reported in [21] have been employed. Relevant parameters in these measurements are cited as follows: 


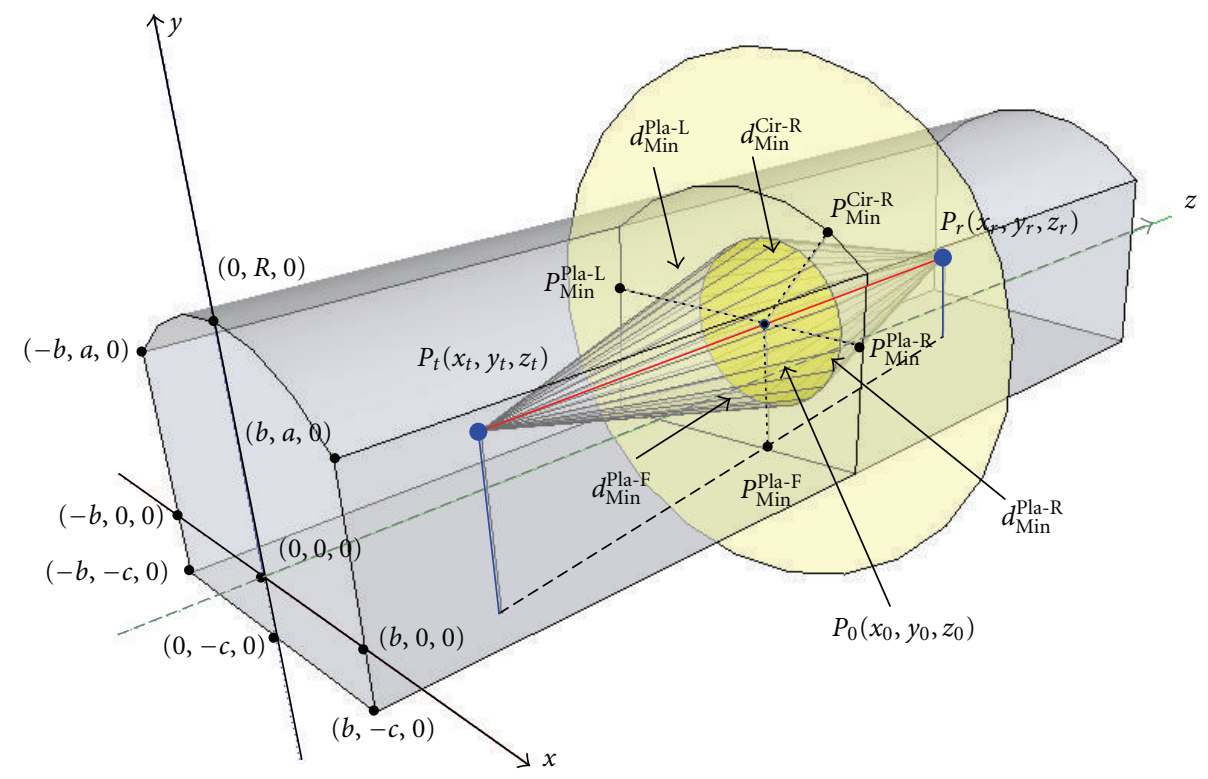

Figure 5: Detailed schematic diagram of the propagation inside the arched tunnel "Type I" with the first Fresnel zone clearance.

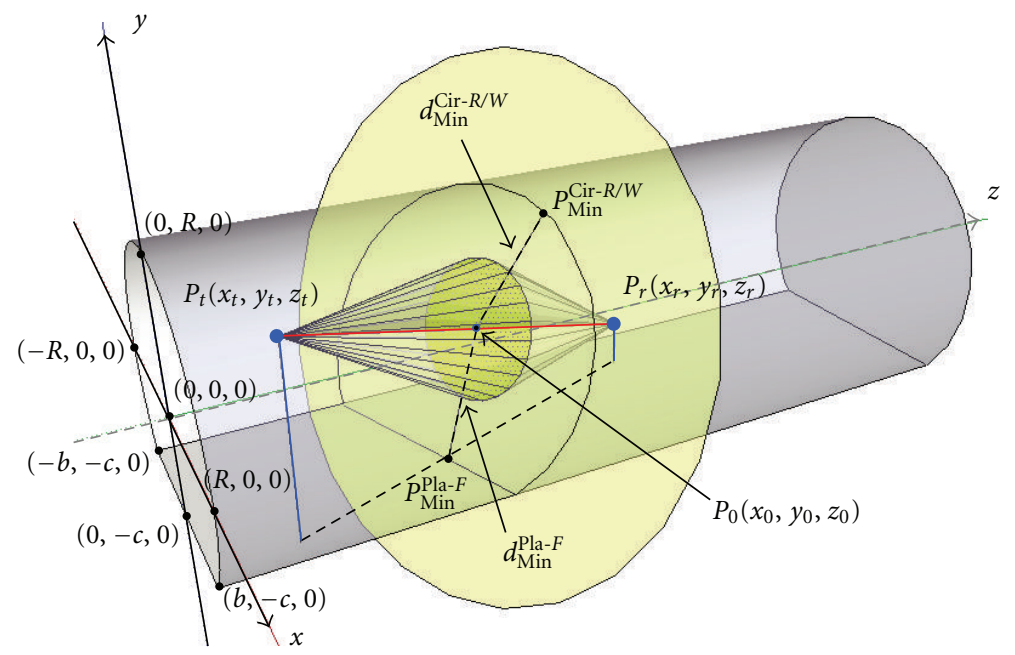

FIGURE 6: Detailed schematic diagram of the propagation inside the arched tunnel "Type II" with the first Fresnel zone clearance.

(i) first group of measurements [21]: $\lambda=0.66, R=4.3$, $x_{t}=1.8, y_{t}=0, z_{t}=0, x_{r}=1.8, y_{r}=0$.

(ii) second group of measurements [21]: $\lambda=0.33, R=$ $4.3, x_{t}=1.8, y_{t}=0, z_{t}=0, x_{r}=1.8, y_{r}=0$.

In the case of the first group, by seeking the simultaneous solution of (12), the minimal positive real root is the coordinate of intersection point with the minimal distance to $P_{0}\left(x_{0}, y_{0}, z_{0}\right)$ in the measured tunnel: $p_{\text {Min }}^{\mathrm{Cir}}(4.3,0,18.94)$. By solving (16) and (17), $z_{r \text { Min }}^{\mathrm{Cir}}=37.88$. Hence, according to (18), the dividing point locates at $z_{r}=37.88$. In term of the second group, $p_{\text {Min }}^{\mathrm{Cir}}(4.3,0,37.88), z_{r_{\text {Min }}}^{\mathrm{Cir}}=75.76$. All the comparison results are shown in Table 1 .

3.3. Dividing Point Model in Arched Tunnel. There are mainly two kinds of arched tunnels. "Type I" consists of three plane walls and an arched roof; “Type II" includes arched walls and roof, but a plane floor, more like a semicircle. Figures 5 and 6 demonstrate the cross-sectional geometry for both types of arched tunnels. It is noteworthy that both the arched tunnel "Type I" and "Type II" can be seen as a combination of a circular tunnel and a rectangular tunnel, but in different configurations. Hence, the dividing point can be modeled in a circular tunnel and a rectangular tunnel independently and then determined by their specific combinations.

Figure 5 shows the propagation inside the arched tunnel "Type I" with the first Fresnel zone clearance. In "Type I", two vertical walls and the floor in the rectangular tunnel as well as the arched roof in the circular tunnel can possibly obstruct the first Fresnel zone; thus, by substituting the functions of the roof, walls, and floor to (6): 
(i) left vertical wall: $x=-b$;

(ii) right vertical wall: $x=b$;

(iii) floor: $y=-c$;

(iv) arched roof: $x^{2}+y^{2}=R^{2},|x| \leq b, a \leq y \leq R$;

$d_{\mathrm{Min}}^{\mathrm{Cir}-R}\left(z_{r}\right), d_{\mathrm{Min}}^{\mathrm{Pla}-R}\left(z_{r}\right), d_{\mathrm{Min}}^{\mathrm{Pla}-L}\left(z_{r}\right)$, and $d_{\mathrm{Min}}^{\mathrm{Pla}-F}\left(z_{r}\right)$ corresponding to the minimal distance from $P_{0}$ to the intersection (line/curve) on the arched roof, the right/left wall and the floor can be obtained. By employing (17), the dividing point location of $z_{r \text { Min }}^{\text {Cir- } R}, z_{r \text { Min }}^{\text {Pla- } R}, z_{r \text { Min }}^{\text {Pla- }}$, and $z_{r \text { Min }}^{\text {Pla- } F}$ corresponding to the touching of the maximum first Fresnel zone and every wall of arched tunnels can be deduced as

$$
\begin{aligned}
& z_{r \text { Min }}^{\mathrm{Cir}-R}=\operatorname{Min}\left\{z_{r} \mid r_{1 \mathrm{Max}}\left(z_{r}\right)=d_{\mathrm{Min}}^{\mathrm{Cir}-R}, z_{r} \in R^{+}\right\}, \\
& z_{r \text { Min }}^{\mathrm{Pla}-R}=\operatorname{Min}\left\{z_{r} \mid r_{1 \text { Max }}\left(z_{r}\right)=d_{\mathrm{Min}}^{\mathrm{Pla}-R}, z_{r} \in R^{+}\right\}, \\
& z_{r \text { Min }}^{\mathrm{Pla}-L}=\operatorname{Min}\left\{z_{r} \mid r_{1 \text { Max }}\left(z_{r}\right)=d_{\mathrm{Min}}^{\mathrm{Pla}-L}, z_{r} \in R^{+}\right\}, \\
& z_{r \text { Min }}^{\mathrm{Pla}-F}=\operatorname{Min}\left\{z_{r} \mid r_{1 \text { Max }}\left(z_{r}\right)=d_{\mathrm{Min}}^{\mathrm{Pla}-F}, z_{r} \in R^{+}\right\} .
\end{aligned}
$$

Thus, the dividing point inside the arched tunnel "Type I" locates at $z_{r}$ :

$$
z_{r}=\operatorname{Min}\left(z_{r \text { Min }}^{\mathrm{Cir}-R}, z_{r \text { Min }}^{\mathrm{Pla}-R}, z_{r \text { Min }}^{\mathrm{Pla}-L}, z_{r \text { Min }}^{\mathrm{Pla}-F}\right) .
$$

Figure 6 illustrates the propagation inside the arched tunnel "Type II" with the first Fresnel zone clearance. In "Type II", only the floor in the rectangular tunnel and the arched roof/wall in the circular tunnel can possibly obstruct the first Fresnel zone; therefore, by substituting the functions of the arched roof and the floor to (6):

(i) floor: $y=-c$;

(ii) arched roof: $x^{2}+y^{2}=R^{2},-c \leq y \leq R$;

$d_{\mathrm{Min}}^{\mathrm{Cir}-R / W}\left(z_{r}\right)$ and $d_{\mathrm{Min}}^{\mathrm{Pla}-F}\left(z_{r}\right)$ corresponding to the minimal distance from $P_{0}$ to the intersection (line/curve) on the arched roof/wall and the floor can be obtained. Then, the dividing point location of $z_{r \mathrm{Min}}^{\mathrm{Cir}-R / W}$ and $z_{r \text { Min }}^{\mathrm{Pla} F}$ corresponding to the touching of the maximum first Fresnel zone and every wall can be derived as

$$
\begin{gathered}
z_{r \text { Min }}^{\mathrm{Cir}-R / W}=\operatorname{Min}\left\{z_{r} \mid r_{1 \mathrm{Max}}\left(z_{r}\right)=d_{\mathrm{Min}}^{\mathrm{Cir}-R}, z_{r} \in R^{+}\right\}, \\
z_{r \text { Min }}^{\mathrm{Pla}-F}=\operatorname{Min}\left\{z_{r} \mid r_{1 \text { Max }}\left(z_{r}\right)=d_{\mathrm{Min}}^{\mathrm{Pla}-F}, z_{r} \in R^{+}\right\} .
\end{gathered}
$$

The dividing point inside the arched tunnel "Type II" locates at $z_{r}$ :

$$
z_{r}=\operatorname{Min}\left(z_{r \operatorname{Min}}^{\operatorname{Cir}-R / W}, z_{r \operatorname{Min}}^{\mathrm{Pla}-F}\right)
$$

Two groups of measurement campaigns have been used for validating the model in arched tunnels.

(i) The first group of received signal strength measurements are performed in a railway tunnel typical to Europe at $400 \mathrm{MHz}$. The tunnel is $520 \mathrm{~m}$ long and originally engineered for a railway, but the line was

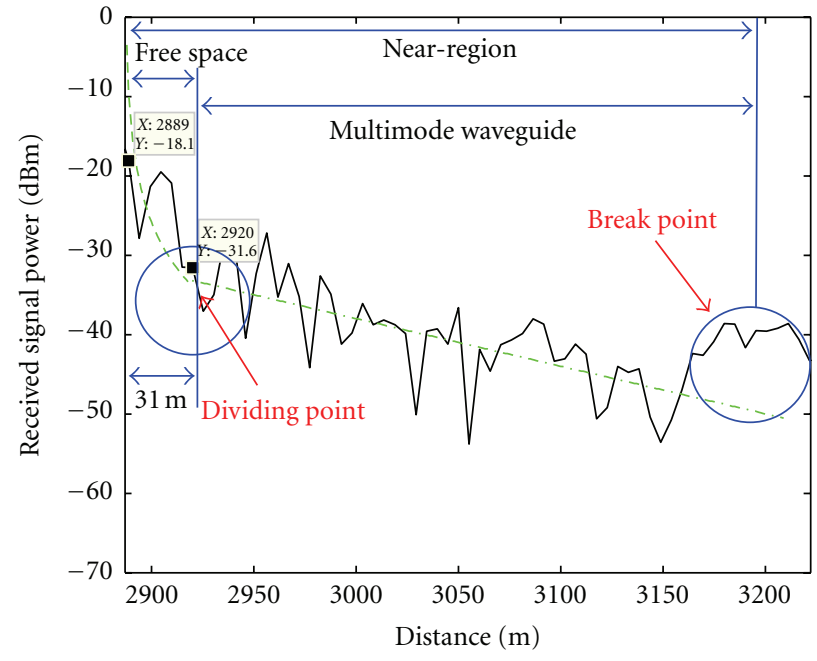

$\begin{array}{ll}\text { _- Measured received signal power } \\ \text { - } & \text { Multi-mode waveguide prediction } \\ \text { - - } & \text { Free space loss model prediction }\end{array}$

Figure 7: Comparisons between measurement and theory on the propagation mechanisms and their dividing point in the nearregion inside an arched tunnel.

closed, and it is now used by pedestrians and cyclists [12]: $\lambda=0.75, R=2.35, x_{t}=0, y_{t}=0, z_{t}=0, x_{r}=$ $0, y_{r}=0, A=0$, and $c=1.5$. By joint solving (12), the coordinate of intersection point with the minimal distance to $P_{0}\left(x_{0}, y_{0}, z_{0}\right)$ in the arched tunnel can be derived: $p_{\text {Min }}^{\mathrm{Cir}-R / W}(0,2.35,13.48), p_{\mathrm{Min}}^{\mathrm{Pla}-F}(0,-1.5,6.85)$. By solving (16) and (17), $z_{r \text { Min }}^{\mathrm{Cir}-R / W}=27, z_{r \text { Min }}^{\mathrm{Pla} F}=$ 13.65. Hence, the dividing point locates at $z_{r}=13.65$.

(ii) The second set of measurements are carried out in a dual carriageway road tunnel linking Austria and Slovenia at $400 \mathrm{MHz}$. The tunnel was closed in one direction at the time, while the second lane operated normally [12]: $\lambda=0.75, R=5.28$, $x_{t}=3.2, y_{t}=-0.8, z_{t}=0, x_{r}=3.2, y_{r}=-0.8$, $c=2.5$. By seeking the simultaneous solution of (12), the coordinate of intersection point with the minimal distance to $P_{0}\left(x_{0}, y_{0}, z_{0}\right)$ can be obtained: $p_{\text {Min }}^{\mathrm{Cir}-R / W}(5.12,-1.28,10.47), \quad p_{\text {Min }}^{\text {Pla-F }}(3.2,-2.5,7.71)$. so, $z_{r \text { Min }}^{\mathrm{Cir}-R / W}=20.94, z_{r_{\text {Min }}}^{\mathrm{Pla}-F}=15.41$. Equation (18) indicates the dividing point locating at $z_{r}=15.41$, which means the Maximum first Fresnel zone first touches the roof of the arched tunnel.

3.4. Validation Results. Table 1 illustrates the global comparisons of the dividing point between the results of model and the measurements inside rectangular, circular, and arched tunnels. The location of the dividing point is extracted from the measurements in the following way: the free space propagation model was compared with the measured received signal power; then, the point, in front of which the fitting is good and behind which is bad, was found. As shown in Table 1, the results indicate that the model for the dividing 


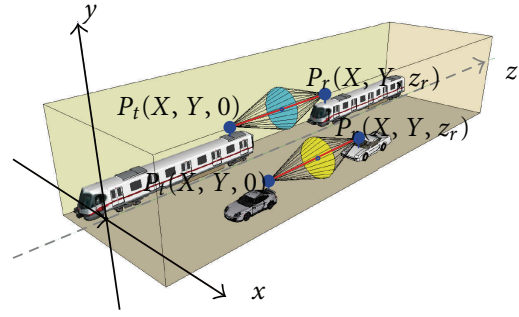

(a)

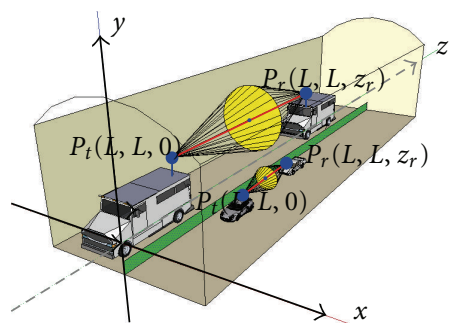

(b)

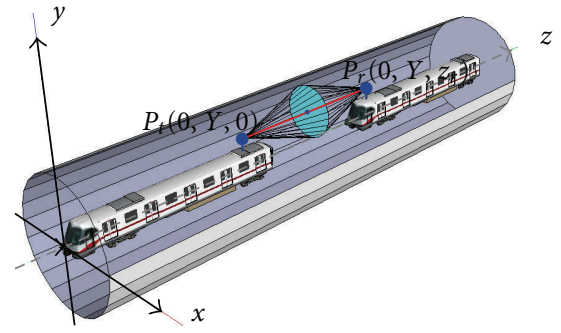

(c)

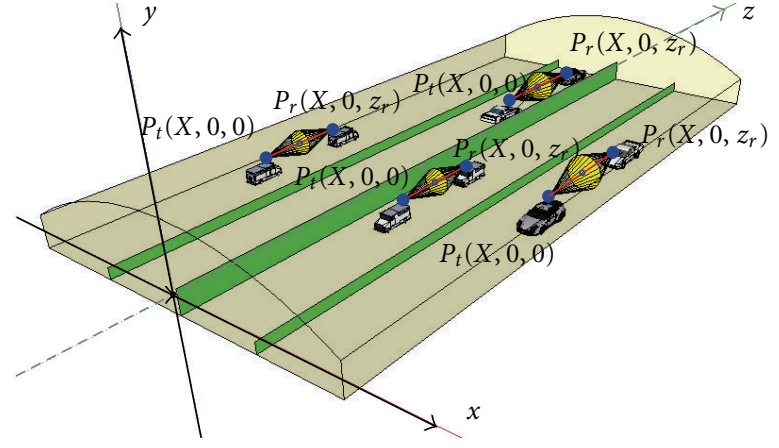

(d)

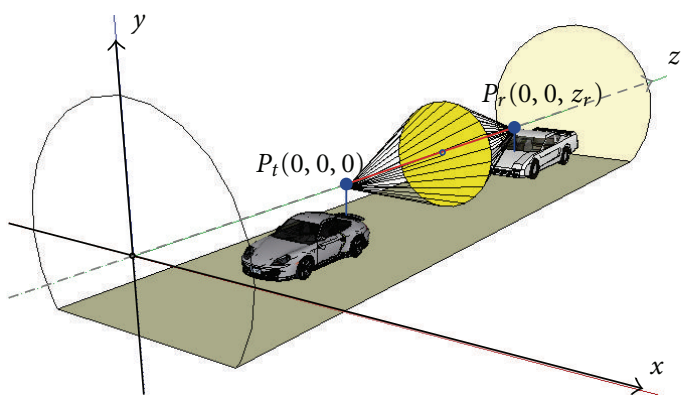

(e)

Figure 8: (a) specific situation one; (b) specific situation two; (c) specific situation three; (d) specific situation Four; (e) specific situation five.

point has a good performance in different types of tunnels at various frequencies.

To clearly depict the effect of the entire propagation model in the near-region of arbitrary cross-sectional tunnels, the measurement carried out in a railway tunnel in Spain at $900 \mathrm{MHz}$ [1] has been employed. As shown in Figure 7, the dividing point separates two propagation segments. The propagation in the segment before the dividing point follows the free space mechanism, and corresponding free space loss model has a good agreement with the measured received signal power. The segment after the dividing point is dominated by the multi-mode waveguide mechanism, and the multimode waveguide loss model shows a good performance as well. The accurate location of the dividing point clearly distinguishes between two propagation mechanisms in the near-region. Therefore, the previous different and seemingly conflicting views $[1,2,6,12-14]$ have been unified by this general model.

All the validation results and comparisons offered above implies that the model for the propagation mechanisms and their dividing point in the near-region are valid and easy to be used in arbitrary cross-sectional tunnels.

\section{Dividing Point Model Simplification and Discussion}

In some real applications, the locations of transmitters and receivers, as well as the motion trajectories of mobile stations follow certain rules. In this section, the simplified formulas of the diving point model in rectangular, circular, and arched tunnels are deduced corresponding to five application situations.

Figure 8(a) illustrates specific situation one: in some systems, such as Dedicated Short-Range Communications (DSRC) [11], the communication is going on between different vehicles (car or carriage). In this case, the transmitter and the receiver always have similar heights and similar tracks: $x_{t}=x_{r}=X, y_{t}=y_{r}=Y$. Figure 8(b) depicts specific situation two, which can be met in DSRC, particularly in the dual carriageway road tunnel where the antennas of transmitter and receiver on vehicle have similar horizontal and vertical distance from the center of the cross-section: $x_{t}=x_{r}=y_{t}=y_{r}=L$. Figure 8(c) shows specific situation three: unlike specific situation two, this situation usually occurs in the one-way narrow tunnel, for both car and train. That means all the communication units move along the central track with similar high antennas: $x_{t}=x_{r}=0, y_{t}=$ $y_{r}=Y$. Figure $8(\mathrm{~d})$ demonstrates specific situation four: like specific situation three, this situation requirements can be met in multi-way wide tunnel, for both cars and trains. In this case, all the communication units move along the same track and the antennas' heights approximately equal the center of the cross-section: $x_{t}=x_{r}=X, y_{t}=y_{r}=$ 0 ; Figure $8(\mathrm{e})$ shows specific situation five: in some long tunnels, especially with the operating frequency of several $\mathrm{GHz}$, the near-region is very long. In this case, by using the modal theory, the transmitter and the receiver can be approximated to be located at the center of the tunnel's crosssection: $x_{t}=x_{r}=0, y_{t}=y_{r}=0$. 
TABLE 2: Simplification of the dividing point model in certain specific situations.

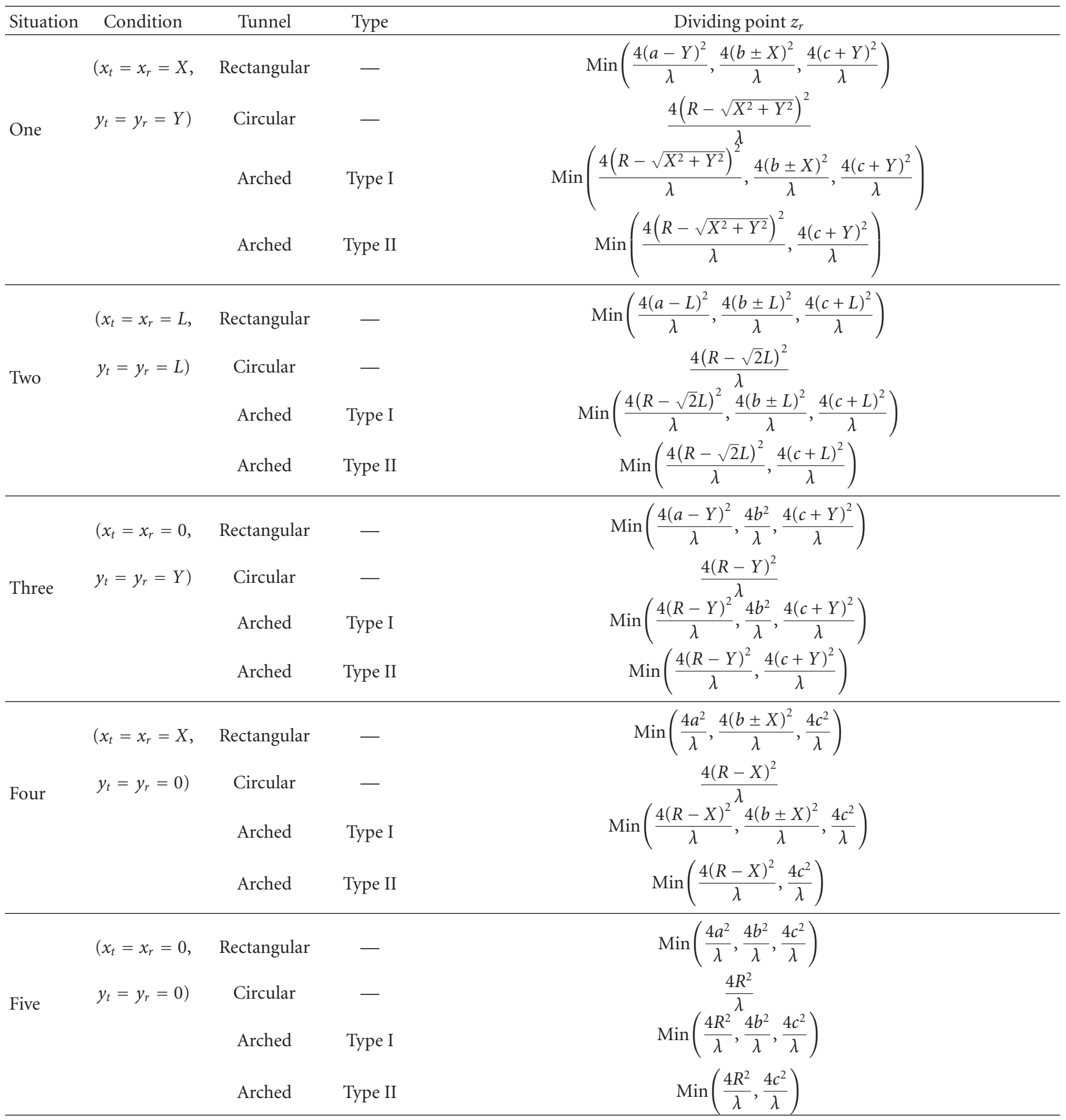

As shown in Table 2, the location of the dividing point in each case can be expressed by simple formulas corresponding to rectangular, circular, and arched tunnels (both "Type I" and "Type II").

All the simplified formulas provide an easy way to determine the location of the areas corresponding to different propagation mechanisms in the near-region, under the realistic application scenarios. Summarizing the general character of the simplified formulas, we have found that the minimal absolute distance between antennas and any of the tunnel surfaces is the dominant factor in the calculation of these cases. This conclusion can be very useful for the system designer to control different mechanism-based propagation areas in the near-region within tunnels. For instance, using this model, new communication based train control system designers can expand or suppress certain propagation mechanisms according their design requirements. 


\section{Conclusion}

This paper clarifies the propagation mechanism situation in the near-region of tunnels. The main contribution of this paper is to present a general analytical approach and model for the dividing point between different propagation mechanisms in arbitrary cross-sectional tunnels for the first time. With the accurate localization of the dividing point, the existing seemingly conflicting views on the propagation in the near-region have been unified. From both the theoretical and measured results in five typical pedestrian, road, and railway tunnels, the dividing point locates from 13.65 to $75.76 \mathrm{~m}$ when the frequency ranges from $400 \mathrm{MHz}$ to $900 \mathrm{MHz}$. This location could be further when the frequency is higher or when the transmitter/receiver is further away from the walls of tunnels.

In order to facilitate the implementation of the proposed model, the specific model in the main types of tunnels (rectangular, circular, and arched tunnels) is deduced. Particularly, in terms of five realistic application situations, the simplified models are given. It has been found that in these cases the minimal absolute distance between the antennas and any of the tunnel surfaces dominates the localization of the dividing point. This conclusion can effectively help system designers to control different mechanismbased propagation areas. The analysis, approach, and model in this paper can be essential and heuristic to a deeper understanding of the propagation mechanism inside tunnel, and can be applied in the realistic radio system design. Future work is to extend the presented model from the straight tunnel to the curved tunnel by considering the influence of the curve.

\section{Acknowledgments}

The authors express their thanks to the supports from the NNSF of China under Grants 60830001 and 61222105, Program for New Century Excellent Talents in University under Grant NCET-09-0206, Beijing NSF 4112048, Fundamental Research Funds for the Central Universities 2010JBZ008, State Key Laboratory of Rail Traffic Control and Safety (RCS2011ZZ008, RCS2010 K008, and RCS2011 K008), and TECRAIL Project of the Polytechnic University of Madrid IPT-2011-1034-370000.

\section{References}

[1] C. Briso-Rodríguez, J. M. Cruz, and J. I. Alonso, "Measurements and modeling of distributed antenna systems in railway tunnels," IEEE Transactions on Vehicular Technology, vol. 56, no. 5, pp. 2870-2879, 2007.

[2] Y. P. Zhang and Y. Hwang, "Enhancement of rectangular tunnel waveguide model," in Proceedings of the Asia-Pacific Microwave Conference (APMC '97), pp. 197-200, December 1997.

[3] Y. P. Zhang and Y. Hwang, "Characterization of UHF radio propagation channels in tunnel environments for microcellular and personal communications," IEEE Transactions on Vehicular Technology, vol. 47, no. 1, pp. 283-296, 1998.
[4] J. Alonso, B. Izquierdo, and J. Romeu, "Break point analysis and modelling in subway tunnels," in Proceedings of the 3rd European Conference on Antennas and Propagation (EuCAP '09), pp. 3254-3258, March 2009.

[5] K. Guan, Z. Zhong, B. Ai, and C. Briso-Rodríguez, "Research of propagation characteristics of break point; Near zone and far zone under operational subway condition," in Proceedings of the 6th International Wireless Communications and Mobile Computing Conference (IWCMC '10), pp. 114-118, July 2010.

[6] Y. P. Zhang, "Novel model for propagation loss prediction in tunnels," IEEE Transactions on Vehicular Technology, vol. 52, no. 5, pp. 1308-1314, 2003.

[7] J. M. Molina-Garcia-Pardo, M. Lienard, A. Nasr, and P. Degauque, "On the possibility of interpreting field variations and polarization in arched tunnels using a model for propagation in rectangular or circular tunnels," IEEE Transactions on Antennas and Propagation, vol. 56, no. 4, pp. 1206-1211, 2008.

[8] ETSI ETR 300-3 ed.1 (2000-02), “Terrestrial Trunked Radio (TETRA), Voice plus Data $(\mathrm{V}+\mathrm{D})$, Designers' guide, Part 3: Direct Mode Operation (DMO)," 2000.

[9] http://www.uic.asso.fr.

[10] "IEEE standard for Communications-Based Train Control (CBTC) performance and functional requirements," 1999.

[11] Notice of Proposed Rulemaking and Order FCC 03-324, Federal Communications Commission, 2003.

[12] A. Hrovat, G. Kandus, and T. Javornik, "Four-slope channel model for path loss prediction in tunnels at $400 \mathrm{MHz}$," IET Microwaves, Antennas and Propagation, vol. 4, no. 5, pp. 571$582,2010$.

[13] Y. P. Zhang, G. X. Zheng, and J. H. Sheng, "Radio propagation at $900 \mathrm{MHz}$ in underground coal mines," IEEE Transactions on Antennas and Propagation, vol. 49, no. 5, pp. 757-762, 2001.

[14] T. Klemenschits and E. Bonek, "Radio coverage of road tunnels at 900 and $1800 \mathrm{MHz}$ by discrete antennas," in Proceedings of the 5th IEEE International Symposium on PIMRC, vol. 2, pp. 411-415, 1994.

[15] J. M. Molina-Garcia-Pardo, M. Lienard, A. Nasr, and P. Degauque, "On the possibility of interpreting field variations and polarization in arched tunnels using a model for propagation in rectangular or circular tunnels," IEEE Transactions on Antennas and Propagation, vol. 56, no. 4, pp. 1206-1211, 2008.

[16] M. Lienard and P. Degauque, "Natural wave propagation in mine environments," IEEE Transactions on Antennas and Propagation, vol. 48, no. 9, pp. 1326-1339, 2000.

[17] P. Mariage, M. Lienard, and P. Degauque, "Theoretical and experimental approach of the propagation of high frequency waves in road tunnels," IEEE Transactions on Antennas and Propagation, vol. 42, no. 1, pp. 75-81, 1994.

[18] S. Saunders, Antennas and Propagation for Wireless Communication Systems, John Wiley and Sons, Chichester, England, 2005.

[19] Z. changsen and M. Yan, "Effects of cross section of mine tunnel on the propagation characteristics of UHF radio wave Antennas," in Proceedings of the 7th International Symposium on Propag. and EM Theory (ISAPE '06), pp. 1-5, 2006.

[20] C. S. Zhang and L. F. Guo, "Research on propagation characteristics of electromagnetic wave in tunnels with arbitrary cross sections," in Proceedings of the 2nd International Conference on Future Computer and Communication (ICFCC '10), pp. V22-V25, May 2010.

[21] D. G. Dudley, M. Liénard, S. F. Mahmoud, and P. Degauque, "Wireless propagation in tunnels," IEEE Antennas and Propagation Magazine, vol. 49, no. 2, pp. 11-26, 2007. 

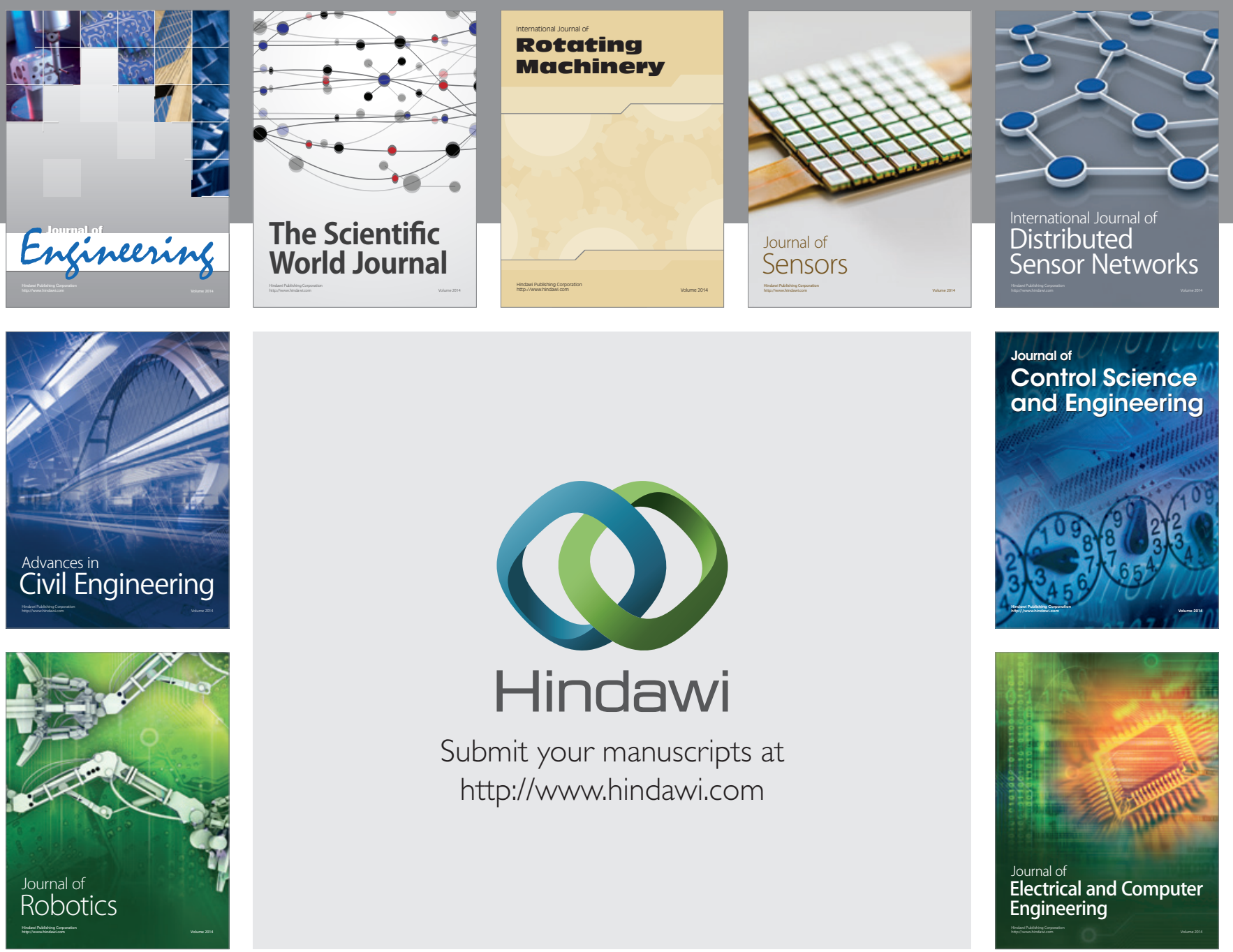

Submit your manuscripts at

http://www.hindawi.com
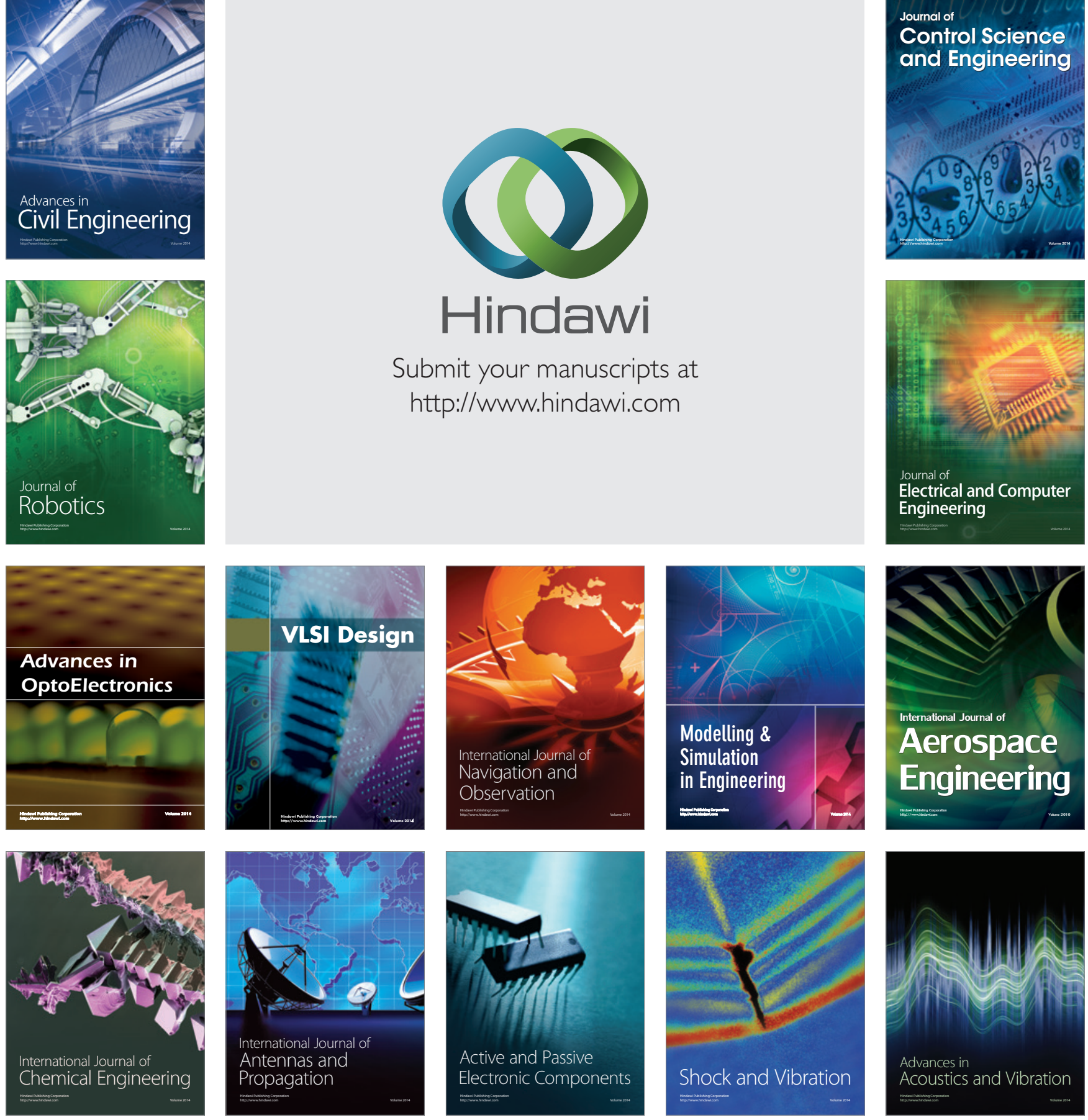